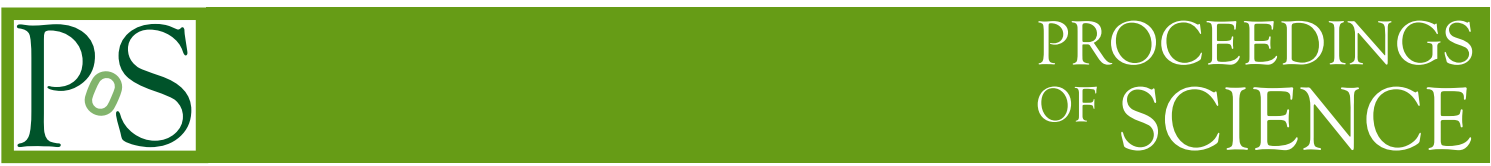

\title{
B meson decays to final states containing charmonia at LHCb
}

\author{
Christian LINN*广 \\ Heidelberg University \\ E-mail: linn@physi.uni-heidelberg.de
}

$\mathrm{LHCb}$ measurements of B mesons containing charmonia in the final state are presented, using data collected during 2010 and 2011. Branching ratios of various $\mathrm{B} \rightarrow \psi(2 \mathrm{~S}) \mathrm{X}$ and $\mathrm{B} \rightarrow \mathrm{J} / \psi \mathrm{X}$ decays are measured. The values of the polarization amplitudes of the decay $\mathrm{B}_{\mathrm{s}}^{0} \rightarrow \mathrm{J} / \psi \overline{\mathrm{K}}^{* 0}$ are determined the for first time. A Dalitz analysis of $\mathrm{B}_{\mathrm{s}}^{0} \rightarrow \mathrm{J} / \psi \pi^{+} \pi^{-}$decays allows the determination of the $\pi^{+} \pi^{-}$resonance structure and the CP content of the decay.

36th International Conference on High Energy Physics

4-11 July 2012

Melbourne, Australia

* Speaker.

$\dagger$ on behalf of the LHCb collaboration 


\section{Introduction}

The LHCb detector is a single-arm forward spectrometer, covering a pseudorapidity of $2<$ $\eta<5$. A full detector description can be found in [1]. Providing a good decay time resolution it is especially designed for measurements of B meson decays. Due to its high muon identification efficiency of $97 \%$ and the good trigger efficiency of $90 \%$ for di-muon channels it can also improve measurements of B decays containing charmonia in the final state. Some of these decays are especially important for $\mathrm{CP}$ violation (CPV) measurements and for probing physics beyond the Standard Model (SM). In the following some results of measurements of B-mesons decaying to charmonia performed at $\mathrm{LHCb}$ are presented.

\section{Branching ratios of $\mathrm{B} \rightarrow \psi(2 \mathrm{~S}) \mathrm{X}$ and $\mathrm{B} \rightarrow \mathrm{J} / \psi \mathrm{X}$ decays}

The measurement of branching ratios of B-meson decays into two-body final sates containing a charmonium resonance is interesting to probe charmonium properties and can open the door for new CPV measurements in the neutral B system. $\mathrm{LHCb}$ has measured the branching ratios of $\mathrm{B}^{+} \rightarrow \psi(2 \mathrm{~S}) \mathrm{K}^{+}$relative to $\mathrm{B}^{+} \rightarrow \mathrm{J} / \psi \mathrm{K}^{+}$, of $\mathrm{B}_{\mathrm{d}}^{0} \rightarrow \psi(2 \mathrm{~S}) \mathrm{K}^{* 0}$ relative to $\mathrm{B}_{\mathrm{d}}^{0} \rightarrow \mathrm{J} / \psi \mathrm{K}^{* 0}$ and of $\mathrm{B}_{\mathrm{s}}^{0} \rightarrow \psi(2 \mathrm{~S}) \phi$ relative to $\mathrm{B}_{\mathrm{s}}^{0} \rightarrow \mathrm{J} / \psi \phi$ [3]. The decays were reconstructed in the decay modes $\psi(2 \mathrm{~S}) \rightarrow \mu^{+} \mu^{-}, \mathrm{J} / \psi \rightarrow \mu^{+} \mu^{-}, \mathrm{K}^{* 0} \rightarrow \mathrm{K}^{+} \pi^{-}$and $\phi \rightarrow \mathrm{K}^{+} \mathrm{K}^{-}$using $0.37 \mathrm{fb}^{-1}$ of data collected at the center-of-mass energy $\sqrt{s}=7 \mathrm{TeV}$. The event yield was determined from maximum likelihood fits to the invariant mass distributions of the selected candidates. After correcting the yield for the detector acceptance and subtracting non-resonant contributions in the $\phi$ and $\mathrm{K}^{* 0}$ modes, the relative branching ratios are measured to be

$$
\begin{aligned}
\frac{\mathrm{BR}\left(\mathrm{B}^{+} \rightarrow \psi(2 \mathrm{~S}) \mathrm{K}^{+}\right)}{\mathrm{B}^{+} \rightarrow \mathrm{J} / \psi \mathrm{K}^{+}} & =0.594 \pm 0.006 \text { (stat.) } \pm 0.016 \text { (syst }) \pm 0.015\left(R_{\psi}\right), \\
\frac{\mathrm{BR}\left(\mathrm{B}_{\mathrm{d}}^{0} \rightarrow \psi(2 \mathrm{~S}) \mathrm{K}^{* 0}\right)}{\mathrm{B}_{\mathrm{d}}^{0} \rightarrow \mathrm{J} / \psi \mathrm{K}^{* 0}} & =0.476 \pm 0.014 \text { (stat.) } \pm 0.010(\text { syst }) \pm 0.012\left(R_{\psi}\right), \\
\frac{\mathrm{BR}\left(\mathrm{B}_{\mathrm{s}}^{0} \rightarrow \psi(2 \mathrm{~S}) \phi\right)}{\mathrm{B}_{\mathrm{s}}^{0} \rightarrow \mathrm{J} / \psi \phi} & =0.489 \pm 0.026 \text { (stat. }) \pm 0.021 \text { (syst) } \pm 0.012\left(R_{\psi}\right),
\end{aligned}
$$

where the third uncertainty is the uncertainty of the relative branching fraction of $\mathrm{J} / \psi$ and $\psi(2 S)$ to $\mu^{+} \mu^{-}$. The systematic uncertainty is dominated by the subtraction of the non-resonant component in the $\mathrm{B}_{\mathrm{d}}^{0}$ and $\mathrm{B}_{\mathrm{s}}^{0}$ decays and by the efficiency correction in the $\mathrm{B}^{+}$decay. The measurements are significantly more precise than the current world average [2].

\section{Branching ratio measurement of $\mathrm{B}_{\mathrm{S}}^{0} \rightarrow \mathrm{J} / \psi \mathrm{K}_{\mathrm{S}}^{0}$}

The $\mathrm{B}_{\mathrm{d}}^{0}-\overline{\mathrm{B}}_{\mathrm{d}}^{0}$ mixing phase $\phi_{\mathrm{d}}$ parameterizes the $\mathrm{CP}$ violation arising from the interference between the direct decay amplitude and and the amplitude for decay after oscillation of the $\mathrm{B}_{\mathrm{d}}^{0}$. The decay $\mathrm{B}_{\mathrm{d}}^{0} \rightarrow \mathrm{J} / \psi \mathrm{K}_{\mathrm{S}}^{0}$ allows for the measurement of $\phi_{\mathrm{d}}$ where the decay amplitude is dominated by tree-level processes. Higher order penguin contributions are suppressed but might become important when the measurement precision increases. $\mathrm{B}_{\mathrm{S}}^{0} \rightarrow \mathrm{J} / \psi \mathrm{K}_{\mathrm{S}}^{0}$ is a promising candidate to estimate the doubly Cabbibo-suppresed higher order corrections to the true decay amplitude [4]. It is the 
$\mathrm{U}$-spin symmetric counter part of $\mathrm{B}_{\mathrm{d}}^{0} \rightarrow \mathrm{J} / \psi \mathrm{K}_{\mathrm{S}}^{0}$. The measurement of the branching fraction of $\mathrm{B}_{\mathrm{s}}^{0} \rightarrow \mathrm{J} / \psi \mathrm{K}_{\mathrm{S}}^{0}$ is a first important step and allows to test the $\mathrm{U}$-spin assumption. This measurement is performed at $\mathrm{LHCb}$ using an integrated luminosity of $0.41 \mathrm{fb}^{-1}$ of data [5]. The $\mathrm{B}_{\mathrm{s}}^{0} \rightarrow \mathrm{J} / \psi \mathrm{K}_{\mathrm{S}}^{0}$ decay is reconstructed in the modes $\mathrm{J} / \psi \rightarrow \mu^{+} \mu^{-}$and $\mathrm{K}_{\mathrm{S}}^{0} \rightarrow \pi^{+} \pi^{-}$where the event sample is divided in two subsamples distinguished by the track types used to reconstruct the $\mathrm{K}_{\mathrm{S}}^{0}$. After an event selection based on a neural network which was trained on part of the measured $\mathrm{B}_{\mathrm{d}}^{0} \rightarrow \mathrm{J} / \psi \mathrm{K}_{\mathrm{S}}^{0}$ signal decays a yield of $72 \pm 11$ and $44 \pm 8 \mathrm{~B}_{\mathrm{S}}^{0} \rightarrow \mathrm{J} / \psi \mathrm{K}_{\mathrm{S}}^{0}$ signal events are observed for the two subsamples. The invariant mass distribution of the selected $\mathrm{B}_{\mathrm{d}}^{0} \rightarrow \mathrm{J} / \psi \mathrm{K}_{\mathrm{S}}^{0}$ and $\mathrm{B}_{\mathrm{S}}^{0} \rightarrow \mathrm{J} / \psi \mathrm{K}_{\mathrm{S}}^{0}$ candidates is shown in figure 1. Relating the measured yield to the measured $\mathrm{B}_{\mathrm{d}}^{0} \rightarrow \mathrm{J} / \psi \mathrm{K}_{\mathrm{S}}^{0}$ yield and correcting for acceptance and efficiency effects the branching ratio of $\mathrm{B}_{\mathrm{s}}^{0} \rightarrow \mathrm{J} / \psi \mathrm{K}_{\mathrm{S}}^{0}$ is determined to be

$\mathrm{BR}\left(\mathrm{B}_{\mathrm{s}}^{0} \rightarrow \mathrm{J} / \psi \mathrm{K}_{\mathrm{S}}^{0}\right)=(1.83 \pm 0.21$ (stat. $) \pm 0.10$ (syst. $\left.) \pm 0.14\left(\frac{\mathrm{f}_{\mathrm{s}}}{\mathrm{f}_{\mathrm{d}}}\right) \pm 0.07\left(\mathrm{BR}\left(\mathrm{B}_{\mathrm{d}}^{0} \rightarrow \mathrm{J} / \psi \mathrm{K}_{\mathrm{S}}^{0}\right)\right)\right) \cdot 10^{-5}$.

The dominant contribution to the systematic uncertainty is the knowledge of the ratio between produced $\mathrm{B}_{\mathrm{s}}^{0}$ and $\mathrm{B}_{\mathrm{d}}^{0}$ mesons, $\frac{f_{s}}{f_{d}}$. The main systematic uncertainties of the measurement are introduced by the choice of the mass probability density function (PDF) used in the determination of the event yields and by the correction for the geometrical detector acceptance. The obtained result is more precise than previous measurements and consistent with theoretical $\mathrm{SU}(3)$ expectations.
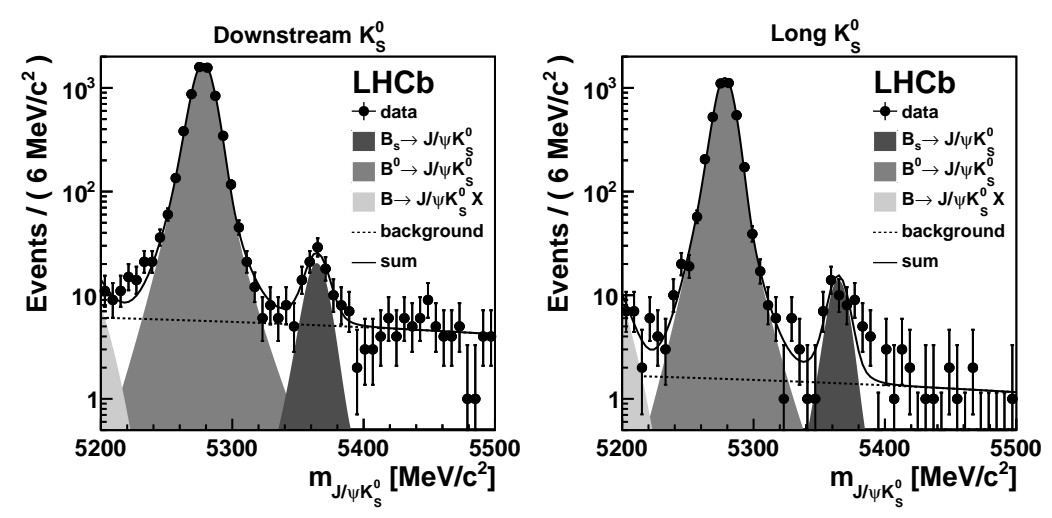

Figure 1: Invariant mass distribution of reconstructed and selected $\mathrm{J} / \psi \mathrm{K}_{\mathrm{S}}^{0}$ candidates. The sample is diveded in two subsample, one not using vertex detector (VeLo) information in the reconstruction of the $\mathrm{K}_{\mathrm{S}}^{0}$ tracks (left) and one using the VeLo information (right).

\section{Resonance structure of $\mathrm{B}_{\mathrm{s}}^{0} \rightarrow \mathrm{J} / \psi \pi^{+} \pi^{-}$decays}

Similar to the mixing-induced $\mathrm{CP}$ violation in the $\mathrm{B}_{\mathrm{d}}^{0}$ sector, the interference of the amplitude of $\mathrm{B}_{\mathrm{s}}^{0}-\overline{\mathrm{B}}_{\mathrm{s}}^{0}$ mixing followed by a decay and the direct decay amplitude into a common $\mathrm{CP}$ eigenstate gives rise to a CP violating phase $\phi_{\mathrm{S}}$ which is important for probing physics beyond the SM. $\phi_{\mathrm{S}}$ was measured at LHCb first using the decay channel $\mathrm{B}_{\mathrm{s}}^{0} \rightarrow \mathrm{J} / \psi \phi[7]$ and then using $\mathrm{B}_{\mathrm{s}}^{0} \rightarrow \mathrm{J} / \psi \mathrm{f}^{0}(980)$ with $\mathrm{f}^{0}(980) \rightarrow \pi^{+} \pi^{-}$[8], which is a pure CP eigenstate in contrast to $\mathrm{B}_{\mathrm{s}}^{0} \rightarrow \mathrm{J} / \psi \phi$. The measurement therefor does not require an angular analysis to separate the different $\mathrm{CP}$ components and is thus complementary to $\mathrm{B}_{\mathrm{s}}^{0} \rightarrow \mathrm{J} / \psi \phi$. Following up on this it is of intrerest to probe the possibility of using a wider $\pi \pi$ mass spectrum and including more final states for the $\phi_{\mathrm{s}}$ measurement. 
To use the decays for the determination of $\phi_{\mathrm{S}}$ it is necessary to determine the CP content of the $\mathrm{B}_{\mathrm{s}}^{0} \rightarrow \mathrm{J} / \psi \pi^{+} \pi^{-}$decays. $1.0 \mathrm{fb}^{-1}$ of data is used to perform a modified Datliz analysis to measure the $\mathrm{B}_{\mathrm{s}}^{0} \rightarrow \mathrm{J} / \psi \pi^{+} \pi^{-}$branching ratio and its resonance structure. The invariant $m_{\mathrm{J} / \psi \pi^{+} \pi^{-}}$mass distribution of all selected events is shown in figure 2, containing $7598 \pm 120$ signal candidates. The probabiltity density functions for the Dalitz analysis describes the square of the invariant masses $m^{2}\left(\mathrm{~J} / \psi \pi^{+}\right), m^{2}\left(\pi^{+} \pi^{-}\right)$and the helicity angle $\theta_{\mathrm{J} / \psi}$ of the $\mathrm{J} / \psi$ for different resonances that could be present in the selected $\pi^{+} \pi^{-}$mass range. The data is fitted with different combinations of interfering resonances plus an additional non-resonant component. The projections of the best fit are shown in figure 3 . A significant presence of the resonances $\mathrm{f}^{0}(980), \mathrm{f}^{0}(1370)$ and $\mathrm{f}^{2}(1270)$ is found. Taking into account their spin structure and the non-resonant decay the fraction of $\mathrm{B}_{\mathrm{s}}^{0} \rightarrow \mathrm{J} / \psi \pi^{+} \pi^{-}$ decays with odd CP eigenstate is determined to be 0.977 at $95 \%$ confidence level. The branching ratio is measured relative to $\mathrm{B}_{\mathrm{s}}^{0} \rightarrow \mathrm{J} / \psi \phi$ as

$$
\frac{\mathrm{BR}\left(\mathrm{B}_{\mathrm{s}}^{0} \rightarrow \mathrm{J} / \psi \mathrm{f}^{0}(980)\right)}{\mathrm{BR}\left(\mathrm{B}_{\mathrm{s}}^{0} \rightarrow \mathrm{J} / \psi \phi\right)}=(21.28 \pm 0.51 \pm 0.56) \%
$$

where the main systematic uncertainties arise from the difference in particle identification efficiency for pions and kaons. More details of this analysis can be found in [9].

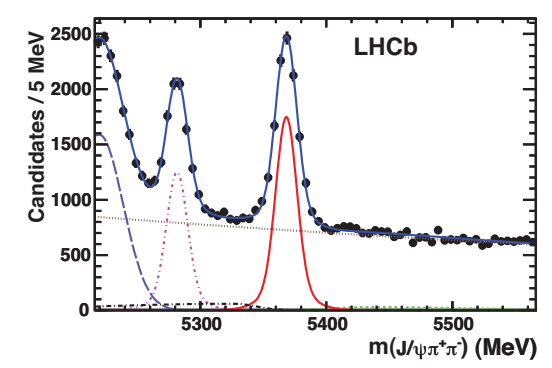

Figure 2: Invariant $m_{\mathrm{J} / \psi \pi^{+} \pi^{-}}$mass distribution of selected $\mathrm{B}_{\mathrm{s}}^{0} \rightarrow \mathrm{J} / \psi \pi^{+} \pi^{-}$candidates. The red solid line shows the signal, the brown dotted line the combinatorial background, the blue dashed line the $\mathrm{B}_{\mathrm{d}}^{0} \rightarrow \mathrm{J} / \psi \mathrm{K} \pi$ reflection background,the green dashed line shows the $\mathrm{B}^{+}$background, the purple dot-dashed represents $\mathrm{B}_{\mathrm{d}}^{0} \rightarrow \mathrm{J} / \psi \pi^{+} \pi^{-}$decays and the black dot-dashed line is a combination of $\mathrm{B}_{\mathrm{s}}^{0} \rightarrow \mathrm{J} / \psi \eta \eta^{\prime}$ and $\mathrm{B}_{\mathrm{s}}^{0} \rightarrow \mathrm{J} / \psi \phi$ with $\phi \rightarrow \pi^{+} \pi^{-} \pi^{0}$ background.

\section{Measurement of the $\mathrm{B}_{\mathrm{s}}^{0} \rightarrow \mathrm{J} / \psi \overline{\mathrm{K}}^{* 0}$ polarization amplitudes}

For the $\mathrm{B}_{\mathrm{s}}^{0} \rightarrow \mathrm{J} / \psi \overline{\mathrm{K}}^{* 0}$ decay the higher order penguin process is not suppresed compared to the tree-level process. Applying SU(3) flavor symmetry arguments, it is a candidate to determine the higher order effects contributing to the measurement of time dependent $\mathrm{CP}$ violation with the decays $\mathrm{B}_{\mathrm{s}}^{0} \rightarrow \mathrm{J} / \psi \phi$ and $\mathrm{B}_{\mathrm{s}}^{0} \rightarrow \mathrm{J} / \psi \mathrm{f}^{0}(980)$ [6]. LHCb performed a measurement of the $\mathrm{B}_{\mathrm{s}}^{0} \rightarrow \mathrm{J} / \psi \overline{\mathrm{K}}^{* 0}$ branching fraction and polarization amplitudes $f_{L}$ and $f_{\|}$that determine the angular properties of the deacy products $\mathrm{J} / \psi \rightarrow \mu^{+} \mu^{-}$and $\mathrm{K}^{* 0} \rightarrow \mathrm{K}^{+} \pi^{-}$. From an integrated luminosity of $0.37 \mathrm{fb}^{-1}$ of pp collisions a sample of $144 \pm 11 \mathrm{~B}_{\mathrm{s}}^{0} \rightarrow \mathrm{J} / \psi \overline{\mathrm{K}}^{* 0}$ signal candidates are selected. The decay $\mathrm{B}_{\mathrm{d}}^{0} \rightarrow \mathrm{J} / \psi \mathrm{K}^{* 0}$ is used as normalization for the branching ration measurement. A unbinned maximum likelihood fit is performed simultaneously to the invariant mass distribution $M_{\mathrm{J} / \psi K \pi}$ and three 


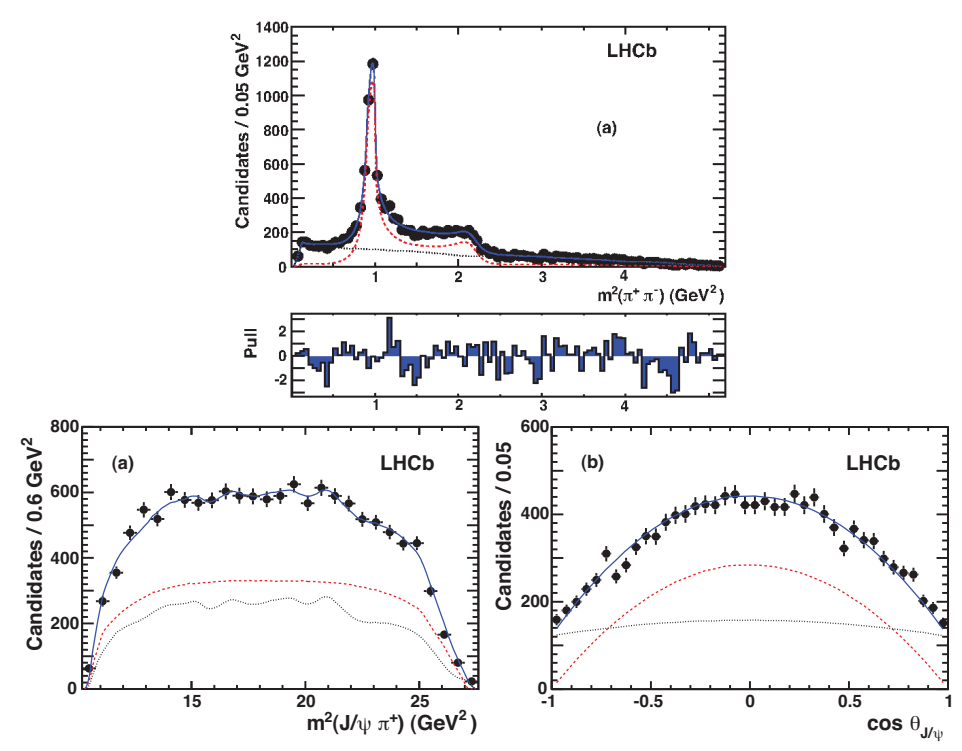

Figure 3: Dalitz fit projections of $m^{2}\left(\mathrm{~J} / \psi \pi^{+}\right), m^{2}\left(\pi^{+} \pi^{-}\right)$and $\theta_{\mathrm{J} / \psi}$. The total fit is represented by the blue solid line and consits of signal (red dashed line) and background (black dotted line)

decay angles $\cos \psi, \cos \theta, \varphi$ of $\mathrm{B}_{\mathrm{s}}^{0} \rightarrow \mathrm{J} / \psi \overline{\mathrm{K}}^{* 0}$ and $\mathrm{B}_{\mathrm{d}}^{0} \rightarrow \mathrm{J} / \psi \mathrm{K}^{* 0}$ to extract the signal yields and the polarization amplitudes. The probabiltity density function describing the angular distributions allows for P- and S-wave components of the $K \pi$ system. Higher relative spin states such as F-wave and $\mathrm{D}$-wave are found to be negligible in the selected $K \pi$ mass window. The one dimensional projections of the fit are shown in figure 4. After correcting the obtained signal yields for the ratio of detection efficiencies, angular acceptances and non-resonant contributions, the branching fraction is determined as

$$
\left.\left.\mathrm{BR}\left(\mathrm{B}_{\mathrm{s}}^{0} \rightarrow \mathrm{J} / \psi \overline{\mathrm{K}}^{* 0}\right)=\left(4.4_{-0.4}^{+0.5} \text { (stat. }\right) \pm 0.8 \text { (syst. }\right)\right) \cdot 10^{-5} .
$$

The systematic uncertainty is dominated by the parameterization of the mass model and the uncertainty of the hadronization fraction $\frac{f_{s}}{f_{d}}$. The result is compatible with a previous CDF measurement [10]. The polarization amplitudes of the $\mathrm{B}_{\mathrm{s}}^{0} \rightarrow \mathrm{J} / \psi \overline{\mathrm{K}}^{* 0}$ decay are measured to be

$$
\begin{gathered}
f_{L}=0.50 \pm 0.08(\text { stat }) \pm 0.02 \text { (syst) } \\
f_{\|}=0.19_{-0.08}^{+0.10}(\text { stat }) \pm 0.02 \text { (syst) } .
\end{gathered}
$$

The dominant systematic uncertainty arise from the angular acceptance correction and the description of the angular distribution of background events. It is the first measurements of the $\mathrm{K}^{* 0}$ polarization fractions in this decay chanel. The polarization fractions of the $\mathrm{B}_{\mathrm{d}}^{0} \rightarrow \mathrm{J} / \psi \mathrm{K}^{* 0}$ were measured as cross-check and agree with previous measurements. More details can be found in [11].

\section{Conclusion}

Some results of LHCb's broad analysis programme with B mesons decaying to charmonia are 

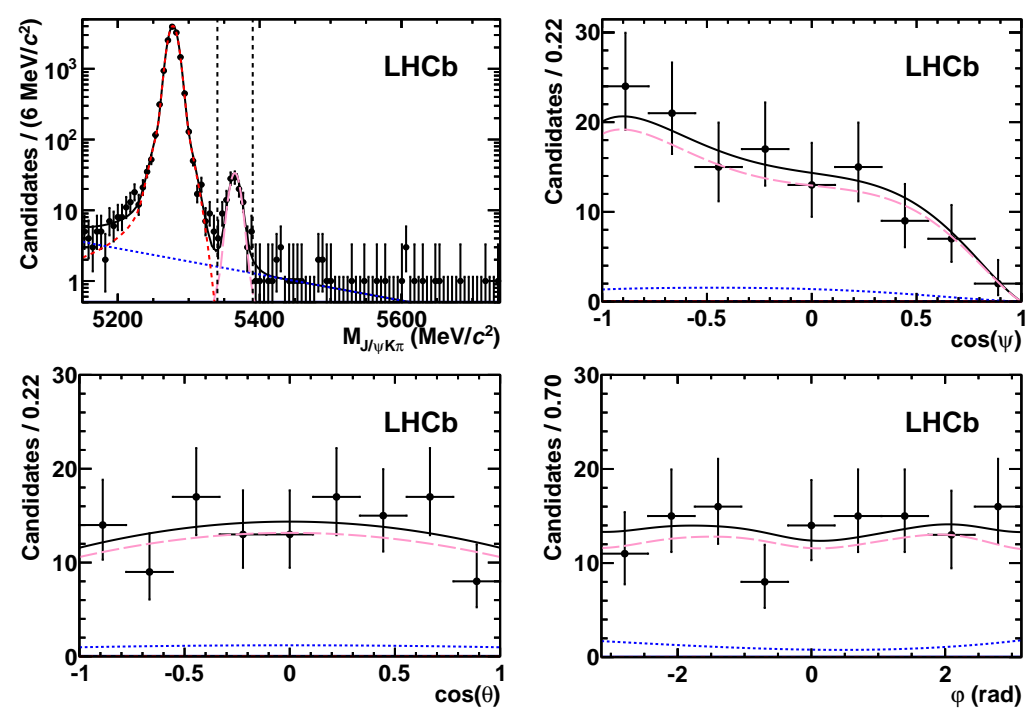

Figure 4: Fit projections on the measured invariant $\mathrm{J} / \psi K \pi$ mass distribution and the decay angles for the mass range indicated by the two dashed vertical lines in the upper right plot. The pink-dashed line is $\mathrm{B}_{\mathrm{s}}^{0} \rightarrow \mathrm{J} / \psi \overline{\mathrm{K}}^{* 0}$ the red dashed line is $\mathrm{B}_{\mathrm{d}}^{0} \rightarrow \mathrm{J} / \psi \mathrm{K}^{* 0}$ and the blued dotted line represents background. The black solid line is their sum.

presented. Branching ratio measurements of $\mathrm{B} \rightarrow \psi(2 \mathrm{~S}) \mathrm{X}$ and $\mathrm{B} \rightarrow \mathrm{J} / \psi \mathrm{X}$ decays were performed with better precision than previous experiments. The polarization amplitudes in $\mathrm{B}_{\mathrm{s}}^{0} \rightarrow \mathrm{J} / \psi \overline{\mathrm{K}}^{* 0}$ decays were measured for the first time. The decomposition of the resonance structure and the determination of the $\mathrm{CP}$ content of $\mathrm{B}_{\mathrm{s}}^{0} \rightarrow \mathrm{J} / \psi \pi^{+} \pi^{-}$decays allows to use the decays for the measurement of mixing-induced $\mathrm{CP}$ violation [12].

\section{References}

[1] A. A. Alves, Jr. et al. (LHCb Collaboration), JINST 3, S08005 (2008).

[2] Particle data Group, K. Nakamura et al., J. Phys. G 37 (2010) 075021.

[3] R. Aaij et al. (LHCb Collaboration), Eur. Phys. J. C 72 (2012) 2118.

[4] R. Fleischer, Eur. Phys. J. C 10 (1999) 299.

[5] R. Aaij et al. (LHCb Collaboration), Phys. Lett. B 713 (2012) 172.

[6] S. Faller, R. Fleischer, T. Mannel, Phys. Rev. D 79, 014005 (2009).

[7] R. Aaij et al. (LHCb Collaboration), Phys. Rev. Lett. 108 (2012) 101803.

[8] R. Aaij et al. (LHCb Collaboration), Phys. Lett. B 707 (2012) 497.

[9] R. Aaij et al. (LHCb Collaboration), Phys. Rev. D 86 (2012) 052006.

[10] T. Aalton et al. (CDF Collaboration), Phys. Rev. D 83, 052012 (2011).

[11] R. Aaij et al. (LHCb Collaboration), Phys. Rev. D 86, 071102(R) (2012).

[12] R. Aaij et al. (LHCb Collaboration), Phys. Lett. B 713 (2012) 378. 